\title{
A hierarchy of electromagnetic gyrokinetic and fluid hybrid models for the simulation of global modes
}

\author{
M. D. J. Cole ${ }^{1}$, A. Mishchenko ${ }^{1}$, A. Könies ${ }^{1}$, R. Hatzky ${ }^{2}$, \\ R. Kleiber ${ }^{1}$ \\ 1 Max Planck Institute for Plasma Physics, D-17491 Greifswald, Germany \\ 2 Max Planck Institute for Plasma Physics, D-85748 Garching, Germany \\ E-mail: michael.cole@ipp.mpg.de
}

\begin{abstract}
.
The goal of magnetic confinement fusion research is to produce an economically viable power reactor. This will require operation at finite plasma $\beta$, where for instance fast particle transport by Alfvén eigenmodes may become important. The density and energy of fast particles originating from heating and fusion will also be higher. Low frequency Alfvénic modes can be destabilised by fast particles, with velocities comparable to the Alfvén velocity, resulting in significant reduction of fast particle confinement. To properly predict and manipulate this behaviour in current and future fusion devices, the numerical simulation of fast particle interaction with Alfvén modes is necessary. Since these simulations are computationally demanding, it is desirable to use the simplest model capable of simulating a given phenomenon. Comparing a case with different physical models also permits the isolation of physics effects responsible for certain phenomena. In this paper we present a hierarchy of such numerical models and investigate their ranges of validity.
\end{abstract}

\section{Introduction}

As the size of tokamak and stellarator fusion devices increases, and methods of confining and controlling high temperature plasmas within them improve, it becomes more pressing to understand high $\beta$ plasma phenomena. The plasma $\beta$ is a measure of the economic efficiency of a reactor and maximising it is important to the viability of magnetic confinement fusion devices as commercial electricity sources.

The importance of fast particle effects will also grow as fusion devices progress towards viable power reactors. Increasing heating requirements will mean greater installed Electron and Ion Cyclotron Resonant Heating (ECRH and ICRH) and Neutral Beam Injection (NBI) power, all of which will contribute to an increased fast particle fraction, especially during startup. Fusion itself will produce high energy (3.5 MeV) alpha particles.

The velocity of fast particles and the Alfvén velocity can be comparable, permitting resonant interaction which can result in unstable modes. Enhanced transport of high energy fast particles could lead to significant localised heat loading on the first wall. Fast particle transport due to interaction with Alfvénic modes has been experimentally observed $[1,2]$.

Since the operating parameters of a viable fusion reactor will differ significantly from those of existing experimental devices, numerical codes are employed in order to 
predict and optimise the behaviour in these devices. A number of models currently exist. One of the most complete is the fully gyrokinetic electromagnetic model, in which the gyrokinetic equation is solved for the bulk electrons, and bulk and fast ions, with motion averaged over the gyrofrequency [3]. Since the timescale of the gyromotion is much shorter than that of the Alfvénic modes, this is an acceptable approximation. Global gyrokinetic codes have been extensively utilised and include for instance the eigenvalue codes LIGKA [4] and KIN-2DEM [5]. This model, however, is highly computationally demanding.

Hybrid gyrokinetic-fluid models have been implemented for a number of codes worldwide. One of the simplest such approaches is to treat the bulk plasma with a Magnetohydrodynamic (MHD) model to compute an Alfvén mode structure, with the fast particles then modelled gyrokinetically, since energy exchange with the mode is a fundamentally kinetic process. In codes such as HMGC, MEGA and M3D [6-8], fast particles interact with the mode structure self-consistently, i.e. the fast particles influence the magnetic and electrostatic field perturbations. Alternatively, fast particle power transfer with a fixed mode can be calculated, as in NOVA-K, CAS3D-K, AE3D-K and VENUS [9-12]. These models neglect bulk kinetic effects, but are very numerically robust. An intermediate model treats the electrons as a fluid, while both the bulk and fast ions are modelled gyrokinetically, an approach that has been used by the codes GEM and GTC $[13,14]$.

All of these models have been implemented for the gyrokinetic code EUTERPE. The standard EUTERPE code models all species gyrokinetically and can treat global modes. The FLU-EUTERPE variant models either the electrons, or the entire bulk plasma, as a fluid, with the fields treated self-consistently, while bulk and fast ions can be modelled gyrokinetically. CKA-EUTERPE couples a mode structure computed by the MHD eigenvalue code CKA with the EUTERPE gyrokinetic model for fast ions, computing the fast particle drive to approximate the growth rate [15].

It is generally advantageous to use the simplest and therefore least resourceintensive model that is capable of capturing the desired physics. Furthermore, comparing the same case with multiple models allows the isolation of physical effects responsible for different phenomena. In this paper, the three models will be outlined and their ranges of validity will be considered. In section 2 , the mathematical details of the models will be set out. In section 3, the applicability of each will be considered in terms of numerical results. We conclude in section 4.

\section{Numerical models}

EUTERPE is a global 3D numerical code that solves the gyrokinetic equation for arbitrary species [16],

$$
\frac{\partial f_{1 s}}{\partial t}+\dot{\mathbf{R}}^{(0)} \cdot \frac{\partial f_{1 s}}{\partial \mathbf{R}}+\dot{v}_{\|}^{(0)} \frac{\partial f_{1 s}}{\partial v_{\|}}=-\dot{\mathbf{R}}^{(1)} \cdot \frac{\partial F_{0 s}}{\partial \mathbf{R}}-\dot{v}_{\|}^{(1)} \frac{\partial F_{0 s}}{\partial v_{\|}},
$$

where subscripts 1 and 0 indicate perturbed and unperturbed quantities respectively, and $s=i, e$, or $f$ indicates the species. The distribution function is split into perturbed and unperturbed components, such that the total distribution function $f_{s}=F_{0 s}+f_{1 s}$. The background distribution functions, $F_{0 s}$, are prescribed, so that solving equation (1) for $f_{1 s}$ gives the distribution function at the next timestep. The unperturbed bulk plasma distribution functions, $F_{0 s}$, are typical represented by 
Maxwellians. For fast particles other choices are also available, such as slowing down distribution functions.

In the PIC method, the perturbed component of the distribution function is discretised with marker particles such that

$$
f_{1 s}\left(\mathbf{R}, v_{\|}, \mu, t\right)=\sum_{\nu=1}^{N_{p}} w_{s \nu}(t) \delta\left(\mathbf{R}-\mathbf{R}_{\nu}\right) \delta\left(v_{\|}-v_{\| \nu}\right) \delta\left(\mu-\mu_{\nu}\right),
$$

where $N_{p}$ is the number of particles, $\nu$ is the particle label and $w_{s \nu}$ is the marker weight. The fields are discretised with finite elements.

\subsection{Fully gyrokinetic 'mixed-variable formulation'}

A key consideration is the choice of formulation of the equations of motion, $\dot{\mathbf{R}}$ and $\dot{v}_{\|}$. In each formulation, these functions in equation (1) are denoted by the same symbols, but take different forms. One approach is to formulate the equations of motion in terms of the canonical parallel momentum, $p_{\|}[3,13]$. This leads to an Ampére's Law of the form

$$
\frac{\beta_{i}}{\rho_{i}^{2}} A_{\|}+\frac{\beta_{e}}{\rho_{e}^{2}} A_{\|}-\nabla_{\perp}^{2} A_{\|}=\mu_{0}\left(\bar{j}_{\| i}+\bar{j}_{\| e}\right),
$$

where $\bar{j}_{\| s}=q_{s} \int \mathrm{d}^{6} Z \delta f_{s} \frac{p_{\|}}{m_{s}} \delta(\mathbf{R}+\boldsymbol{\rho}-\mathbf{x})$ is the perturbed gyrocentre current (distinct from the physical current), the gyrokinetic phase-space volume $\mathrm{d}^{6} Z=$ $B_{\|}^{*} \mathrm{~d} \mathbf{R} \mathrm{d} v_{\|} \mathrm{d} \mu \mathrm{d} \theta$, the thermal gyroradius $\rho_{s}=\sqrt{m_{s} T_{s}} /(e B)$ and $\beta_{s}=\mu_{0} n_{0} T_{s} / B_{0}^{2}$. $\mu_{0}$ is the permeability of free space, $n_{0}$ is the unperturbed density, $T_{s}$ the species temperature, and $B_{0}$ the magnetic field on axis.

The two 'skin terms' in terms of $\beta$ on the left are generated by the choice of co-ordinates, and exist only to cancel other such contributions to the perturbed gyrocentre current on the right. A small relative error in these large co-ordiante generated terms can therefore result in a large relative error in the small physically relevant term, $\nabla_{\perp}^{2} A_{\|}$. The problem is therefore particularly severe at low $k_{\perp}$, at finite plasma $\beta$, and, since $\beta_{s} / \rho_{s}^{2}$ scales inversely with the species mass, when treating electrons. Any small numerical inconsistencies present in a gyrokinetic code can be amplified by the cancellation problem. Work has been done to mitigate many of these inconsistencies, for instance the different discretisation schemes used for $A_{\|}$(finite elements) and $\bar{j}_{\|}$(markers) [17-19].

Despite these efforts, the problem remained prohibitive in many cases at low $k_{\|}$and in 3D geometries. The problem can be further mitigated by choice of appropriate variables, the 'mixed-variable formulation'. In this formulation the perturbed equations of motion are as follows,

$$
\begin{aligned}
\dot{\mathbf{R}}^{(1)} & =\frac{\mathbf{b}}{B_{\|}^{*}} \times \nabla\left\langle\phi-v_{\|} A_{\|}^{(\mathrm{s})}-v_{\|} A_{\|}^{(\mathrm{h})}\right\rangle-\frac{q}{m}\left\langle A_{\|}^{(\mathrm{h})}\right\rangle \mathbf{b}^{*} \\
\dot{v}_{\|}^{(1)} & =-\frac{q}{m}\left[\mathbf{b}^{*} \cdot \nabla\left\langle\phi-v_{\|} A_{\|}^{(\mathrm{h})}\right\rangle+\frac{\partial}{\partial t}\left\langle A_{\|}^{(\mathrm{s})}\right\rangle\right] \\
-\frac{\mu}{m} & \frac{\mathbf{b} \times \nabla B}{B_{\|}^{*}} \cdot \nabla\left\langle A_{\|}^{(\mathrm{s})}\right\rangle,
\end{aligned}
$$


A hierarchy of electromagnetic gyrokinetic and fluid hybrid models for the simulation of global modes 4

$$
\begin{aligned}
\mathbf{b}^{*} & =\frac{\mathbf{B}}{\tilde{B}_{\|}^{*}}+\frac{m_{s}}{\tilde{B}_{\|}^{*} q_{s}} v_{\|}(\nabla \times \mathbf{b}), \\
\tilde{B}_{\|}^{*} & =B+\mathbf{b} \cdot \frac{m_{s}}{q_{s}} v_{\|}(\nabla \times \mathbf{b})+\mathbf{b} \cdot \nabla \times\left\langle A_{\|}^{(s)}\right\rangle \mathbf{b},
\end{aligned}
$$

$q_{s}$ is the species charge, $m_{s}$ is the species mass and $\phi$ is the perturbed electrostatic potential. The gyroaverages are defined as usual. The perturbed magnetic potential, $A_{\|}$, is split into 'symplectic' and 'hamiltonian' parts:

$$
A_{\|}=A_{\|}^{(\mathrm{s})}+A_{\|}^{(\mathrm{h})}
$$

The significance of these two components is related to the formulation of the gyrokinetic Lagrangian from which the equations of motion are derived, and is discussed in more detail in [20,21].

In the mixed-variable formulation, $v_{\|}$is not the actual guiding centre velocity, but rather

$$
v_{\|}=v_{\|}^{(\mathrm{gc})}+\frac{\mathbf{b}^{*}}{B} \cdot \nabla\left[\int^{\theta_{(\mathrm{gc})}}(\psi-\langle\psi\rangle) \mathrm{d} \theta_{(\mathrm{gc})}\right]+\frac{e}{m} A_{\|}^{(\mathrm{h})}+\frac{e}{m} \widetilde{A}_{\|}^{(\mathrm{s})}
$$

where

$$
\psi=\phi-v_{\|}^{(\mathrm{gc})} A_{\|}, \quad \widetilde{A}_{\|}^{(\mathrm{s})}=A_{\|}^{(\mathrm{s})}-\left\langle A_{\|}^{(\mathrm{s})}\right\rangle,
$$

with $v_{\|}^{(g c)}$ being the actual guiding centre velocity.

The system of equations is completed by the quasineutrality equation and Ampére's Law, in the mixed-variable formulation as follows,

$$
\begin{aligned}
& \int \frac{q_{i} F_{0 i}}{T_{i}}(\phi-\langle\phi\rangle) \delta(\mathbf{R}+\boldsymbol{\rho}-\mathbf{x}) \mathrm{d}^{6} Z=\bar{n}_{1 i}-\bar{n}_{1 e} \\
& \left(\frac{\beta_{i}}{\rho_{i}^{2}}+\frac{\beta_{e}}{\rho_{e}^{2}}-\nabla_{\perp}^{2}\right) A_{\|}^{(\mathrm{h})}-\nabla_{\perp}^{2} A_{\|}^{(\mathrm{s})}=\mu_{0}\left(\bar{j}_{\| 1 i}+\bar{j}_{\| 1 e}\right)
\end{aligned}
$$

where the mixed-variable gyrocentre density $\bar{n}_{1 s}=\int \mathrm{d}^{6} Z f_{1 s}^{(\mathrm{m})} \delta(\mathbf{R}+\boldsymbol{\rho}-\mathbf{x})$ and mixedvariable gyrocentre current, $\bar{j}_{\| 1 s}=q_{s} \int \mathrm{d}^{6} Z f_{1 s}^{(\mathrm{m})} v_{\|} \delta(\mathbf{R}+\boldsymbol{\rho}-\mathbf{x})$. Note that these are not the same as the physical density and current.

In the case of finite $A_{\|}^{(h)}$, the 'skin terms' reappear, along with the cancellation problem. However, $A_{\|}^{(s)}$ and $A_{\|}^{(h)}$ can be assigned arbitrarily provided that the physical quantity $A_{\|}$remains constant, the difference being accounted for by the equations of motion. We therefore choose a scheme that minimises $A_{\|}^{(h)}$.

At each timestep, we set $A_{\|}^{(s)}=A_{\|}$(i.e. $A_{\|}^{(h)}=0$ ). Consistency demands that the distribution function also be modified to take account of this operation. Since the symplectic distribution function is independent of the distribution of $A_{\|}$between its two components, and we are setting $A_{\|}=A_{\|}^{(s)}$, we can simply set the mixed-variable formulation distribution function to its symplectic counterpart,

$$
f_{1 s(\text { new })}^{(\mathrm{m})}\left(t_{i}\right)=f_{1 s}^{(\mathrm{s})}\left(t_{i}\right)=f_{1 s(\mathrm{old})}^{(\mathrm{m})}\left(t_{i}\right)+\frac{q_{s}\left\langle A_{\| \text {(old })}^{(\mathrm{h})}\left(t_{i}\right)\right\rangle}{m_{s}} \frac{\partial F_{0 s}}{\partial v_{\|}}
$$


and within each timestep define $A_{\|}^{(s)}$ by the Ohm's law-like relation,

$$
\frac{\partial A_{\|}^{(s)}}{\partial t}+\nabla_{\|} \phi=0 \text {. }
$$

When equation (14) produces an $A_{\|}^{(s)}$ evolution that closely approximates that of the total $A_{\|}$, the accumulation of $A_{\|}^{(h)}$ is reduced, diminishing the relative importance of the skin terms in equation (12). This mitigates the cancellation problem. This approach depends on the choice of a physically accurate form of Ohm's law, suggesting that the scheme may limit $E_{\|}$physics; in section 3 it is shown numerically that this is not the case.

Recalling that the case where $A_{\|}^{(h)}=A_{\|}, A_{\|}^{(s)}=0$ corresponds to the conventional $p_{\|}$-formulation, and referring to equation (5), one can see how the mixed-variable formulation alters the structure of the equations. The relation (14) results in the cancellation of the two terms $\partial\left\langle A_{\|}^{(\mathrm{s})}\right\rangle / \partial t$ and $\mathbf{b} \cdot \nabla \phi$. Since $A_{\|}^{(h)}$ is furthermore set to zero at each timestep, the entire first term in square brackets on the right hand side of equation (5), which scales inversely with the species mass, is greatly diminished in magnitude. The second term, which exhibits no scaling with $m_{s}$, is otherwise generally much smaller. Notice that in equation (12), the key term $\beta_{s} / \rho_{s}^{2}$ exhibits the same $1 / m_{s}$ scaling. In this way, the parallel dynamics of the marker particles are dramatically simplified.

\subsection{Electron fluid model - FLU-EUTERPE}

Another approach is to avoid the cancellation problem entirely by formulating the equations of motion in terms of physical $v_{\|}$. In this formulation the equations of motion are

$$
\dot{\mathbf{R}}=v_{\|} \tilde{\mathbf{b}}^{*}+\frac{1}{q_{s} \tilde{B}_{\|}^{*}} \mathbf{b} \times\left[\mu \nabla B+q_{s}\left(\nabla\langle\phi\rangle+\frac{\partial\left\langle A_{\|}\right\rangle}{\partial t} \mathbf{b}\right)\right]
$$

and

$$
\dot{v}_{\|}=-\frac{1}{m_{s}} \tilde{\mathbf{b}}^{*} \cdot \mu \nabla B-\frac{q_{s}}{m_{s}}\left(\tilde{\mathbf{b}}^{*} \cdot \nabla\langle\phi\rangle+\frac{\partial\left\langle A_{\|}\right\rangle}{\partial t}\right),
$$

where

$$
\tilde{\mathbf{b}}^{*}=\mathbf{b}^{*}+\frac{\nabla \times\left\langle A_{\|}\right\rangle \mathbf{b}}{\tilde{B}_{\|}^{*}}
$$

and $B_{\|}^{*}$ is as in equation (7), substituting $A_{\|}$for $A_{\|}^{(s)}$. The perturbed density and current are defined as

$$
n_{1 s}=\int d^{6} Z f_{1 s} \delta(\mathbf{R}+\boldsymbol{\rho}-\boldsymbol{x}), \quad j_{\| 1 s}=q_{s} \int d^{6} Z f_{1 s} v_{\|} \delta(\mathbf{R}+\boldsymbol{\rho}-\boldsymbol{x}) .
$$

In this formulation, the cancellation problem does not appear in Ampere's law, but the numerically unfavourable [22] time derivative $\partial\left\langle A_{\|}\right\rangle / \partial t$ would need to be solved in (15) and (16) using computationally intensive implicit methods. This can be avoided by defining an Ohm's law as in the mixed-variable formulation, except this time limiting the physics of the model. This can be achieved in a reduced fluid model, 
where the total $A_{\|}$is used in equation (14). The simplest approach is to consider ideal MHD,

$$
E_{\|}=-\nabla_{\|} \phi-\frac{\partial A_{\|}}{\partial t}=0
$$

with a continuity equation for the perturbed electron density derived as a zeroth order moment of the drift kinetic equation. We assume pressure isotropy, $P_{1 e}=P_{\| 1 e}=P_{\perp 1 e}$, and ignore non-linear terms, to arrive at the following equation,

$$
\begin{aligned}
& \frac{\partial n_{1 e}}{\partial t}+n_{0} \mathbf{B} \cdot \nabla\left(\frac{u_{\| 1 e}}{B}\right)+B \mathbf{v}_{E} \cdot \nabla\left(\frac{n_{0}}{B}\right)+\left(\nabla \times A_{\|} \mathbf{b}\right) \cdot \nabla\left(\frac{n_{0} u_{\| 0 e}}{B}\right) \\
& +n_{0}\left(\mathbf{v}_{*}-\mathbf{v}_{E}\right) \cdot \frac{\nabla B}{B}+\frac{\nabla \times \mathbf{B}}{B^{2}} \cdot\left[-\frac{\nabla P_{1 e}}{e}+n_{0} \nabla \phi\right]=0
\end{aligned}
$$

where

$$
\mathbf{v}_{E}=\frac{\mathbf{b} \times \nabla \phi}{B}, \quad \mathbf{v}_{*}=2 \frac{\mathbf{b} \times \nabla P_{1 e}}{n_{0} m_{e} e B}, \quad \frac{\partial P_{1 e}}{\partial t}=-\vec{v}_{E} \cdot \nabla P_{0} .
$$

This model is more robust than the fully gyrokinetic formulation, entirely eliminating the cancellation problem in all circumstances and reducing computational requirements in general. However, the physics content is limited by the pressure and parallel electric field truncations, and electron kinetic effects are neglected entirely. Gyrokinetic bulk ion contributions can also be neglected, using (20) to derive an equation for the total charge density. It is possible in this way to treat the entire bulk plasma as a single fluid, while modelling fast ions gyrokinetically [23]. Stepwise expansion of the physics included in the fluid truncations is possible.

\subsection{Perturbative hybrid model}

The most robust gyrokinetic model for global modes eliminates self-consistent field evolution entirely. A mode structure (i.e. electrostatic and magnetic potentials) is calculated using a pure MHD code, in this case the eigenvalue code CKA [24], which solves the reduced ideal MHD vorticity equation,

$$
\begin{aligned}
& \omega^{2} \nabla \cdot\left(\frac{1}{v_{A}^{2}} \nabla_{\perp} \phi\right)+\nabla \cdot\left[\mathbf{b} \nabla_{\perp}^{2}(\mathbf{b} \cdot \nabla) \phi\right]-\nabla \cdot\left(\frac{\mu_{0} j_{\| 0}}{B}[\nabla \times(\boldsymbol{b}(\boldsymbol{b} \cdot \nabla \phi))]_{\perp}\right) \\
& -\nabla \cdot\left(\frac{2 \mu_{0}}{B^{2}}\left[(\mathbf{b} \times \nabla \phi) \cdot \nabla P_{0}\right](\mathbf{b} \times \boldsymbol{\kappa})\right)=0 .
\end{aligned}
$$

This mode structure is then used as a fixed background to which gyrokinetic fast particles are applied. Measuring the power transfer between the particles and the mode allows us to estimate the growth rate of the mode. This is the approach used in CKA-EUTERPE [15]. Since a desired mode can be selected, rather than having to evolve self-consistently as the fastest growing of many modes, this approach is very numerically robust and flexible, at the expense of limited physics content. An important limitation is that, because the perturbed fields loaded from CKA do not respond to the fast particles in EUTERPE, this model does not account for effects on the growth rate or mode frequency due to modification of the MHD mode structure. In particular, Energetic Particle Modes (EPMs) are beyond the scope of this model. 

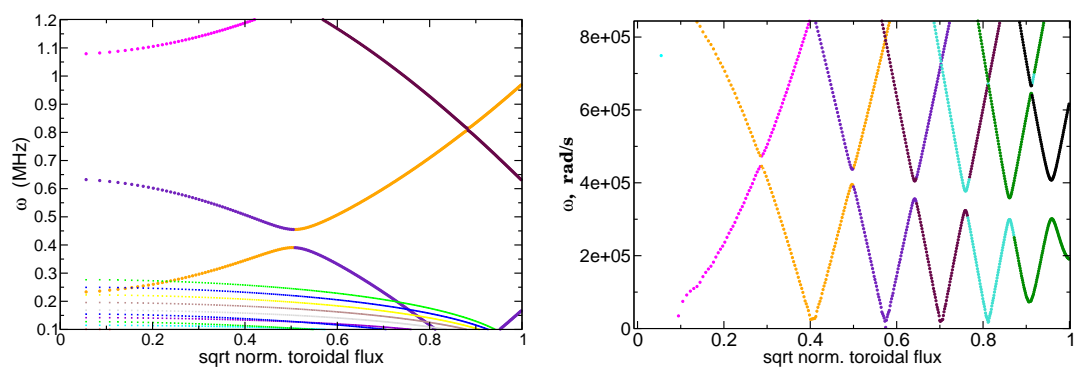

Figure 1. The Alfvén spectra for the low shear ITPA case (left) and the corresponding high shear case (right).

\section{Numerical results}

One phenomenon of particular interest is the Toroidal Alfvén Eigenmode (TAE). TAEs have been observed in many devices, and can be destabilised by fast particles. In a simple case, such as the ITPA TAE benchmark [24], the mode is not strongly affected by mode structure modification due to fast particles, bulk plasma kinetics, or finite $E_{\|}$. In such a case, reduced models may be employed effectively. In this case, which has been run by many codes worldwide, the TAE is simulated in a large aspect ratio tokamak with major radius $R_{0}=10.0 \mathrm{~m}$, minor radius $r_{a}=1.0 \mathrm{~m}$, and magnetic field strength $B_{0}=3.0 \mathrm{~T}$. The background plasma profiles are flat with density $n_{0}=2 \times 10^{19} \mathrm{~m}^{-3}$ and ion and electron temperatures $T_{i}=T_{e}=1 \mathrm{keV}$. The bulk plasma may be treated as a fluid, but here we treat the bulk ions gyrokinetically, and the bulk electrons as a fluid. The safety factor profile $q(r)=1.71+0.16\left(r / r_{a}\right)^{2}$. The spectrum is shown in figure 1 (left).

A Maxwellian distribution of fast deuterium ions is modelled gyrokinetically. The fast particle density is given by the expression

$$
n_{f}(s)=n_{0 f} \exp \left[-\frac{\Delta_{n f}}{L_{n f}} \tanh \left(\frac{s-s_{n f}}{\Delta_{n f}}\right)\right],
$$

where $s$ is the square root of the normalised poloidal flux, the position of maximal logarithmic density gradient $s_{n f}=0.5$, the density at the $s_{n f}$ position $n_{0 f}=$ $0.75 \times 10^{17} \mathrm{~m}^{-3}$, the scale width of the density profile $\Delta_{n f}=0.2$, the scale length of the density profile $L_{n f}=0.3$. The fast ion temperature profile is flat, with the temperature $T_{f}$ being varied between $100 \mathrm{keV}$ and $800 \mathrm{keV}$. The markers are initially loaded uniformly distributed in velocity space.

The TAE is localised around $s=0.5$, excited by fast particles, and exhibits a characteristic frequency situated within the continium gap. The results of the scan over fast particle temperature are compared to those obtained with the fully gyrokinetic code EUTERPE.

This case can be considered by all three models. In figure 2 (left) we compare the measured growth rate of the TAE as it varies with fast particle temperature for each. There is little divergence between them, and so we conclude that in these simple TAE cases even the simplest model is sufficient. This behaviour had previously been considered both analytically [25,26] and numerically [27].

In general, TAE activity can be substantially more complex. Keeping all device parameters the same, and altering only the safety factor profile to $q(r)=1.5+\left(r / r_{\mathrm{a}}\right)^{2}$, 

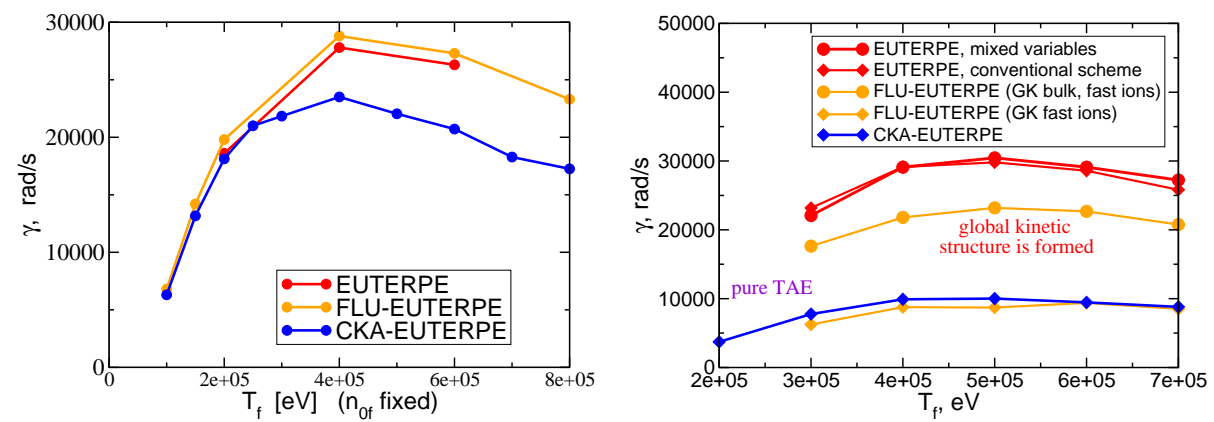

Figure 2. Comparison of growth rates obtained with EUTERPE, FLUEUTERPE, and CKA-EUTERPE for the ITPA TAE benchmark case (left) and for the corresponding high shear case (right). In the high shear case, the fully gyrokinetic EUTERPE is considered with the new mixed variables and conventional $p_{\|}$formulations of the equations of motion, while the fluidgyrokinetic hybrid model is considered treating both bulk electrons and the entire bulk plasma as a fluid. In the ITPA TAE case, the fluid electron model and $p_{\|}$ formulation is used; in this case these distinctions do not affect the results.

a significantly more complex spectrum results (see figure 1, right), in which Alfvén eigenmode activity is correspondingly more complex. Raising the bulk ion temperature from $1 \mathrm{keV}$ to $9 \mathrm{keV}$, the TAE frequency increases such that the mode interacts with the continuum.

This case provides a powerful tool for distinguishing between the models. Referring to figure 2, we can see that the trend in growth rate increases stepwise with the physical completeness of the model. The perturbative hybrid model, CKAEUTERPE, has a fixed mode structure and does not take account of any bulk plasma kinetic effects. As such, it does not correctly account for the mode interaction with the continuum. The growth rate is the same as in the case where $1 \mathrm{keV}$ and the mode frequency lies in the gap; see [28] for further details.

The hybrid model FLU-EUTERPE can treat either the entire bulk plasma or just the bulk electrons as a fluid. The former model is less complete, and the linear growth rates agree with the simpler perturbative hybrid approach. In the latter, more complete, model, we see consistently higher growth rates. This suggests that ion gyrokinetics are important in driving the mode in this case. Comparing the mode structures in figure 3, we see that there is a significant difference, with the global gyrokinetic structure [28] not present without kinetic bulk ions. Continuum damping, which is not present without ion kinetic effects, serves to eliminate the modes at the crossing points. However, although the growth rate shows much closer quantitative agreement with the full gyrokinetic model, it is still consistently underpredicted, possibly owing to the parallel force balance $E_{\|}=0$ truncation.

Finally, this case is used to compare the two fully gyrokinetic schemes: the new mixed variables formulation and the previous, $p_{\|}$, formulation of the equations of motion. Since this case is non-ideal, with a significant finite $E_{\|}$contribution, it serves to demonstrate that the mixed varibles scheme is complete and not truncated by the approximation in (14).

Another problem of interest is the simulation of global modes in stellarators. Since the cancellation problem has hitherto been particularly severe in 3D geometries, 

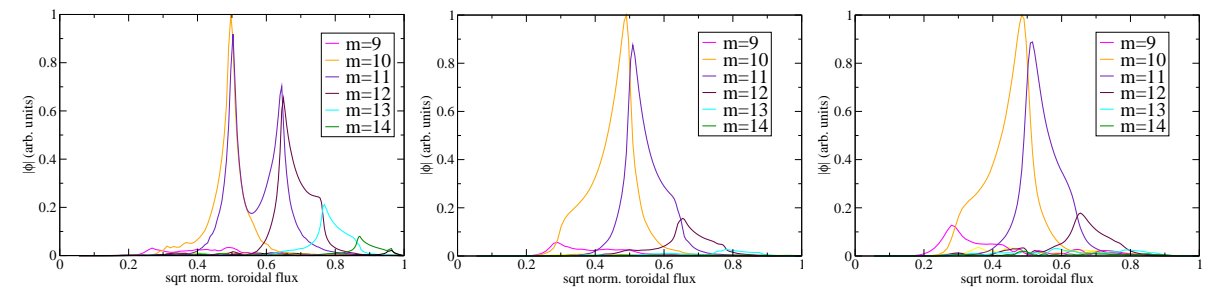

Figure 3. Mode structures plotted for the high shear TAE obtained using (left to right) the fluid hybrid model with gyrokinetic fast ions only, the fluid hybrid model with gyrokinetic bulk and fast ions, and the fully gyrokinetic model. Note that in the case without gyrokinetic bulk ions, continuum damping is not present and TAE modes at all crossing points are visible. The side structures, which may be important in driving the mode [28], are more prominent in the fully gyrokinetic case.

this problem had previously only been approached with the reduced model CKAEUTERPE [29]. The mixed variables scheme greatly mitigates this problem, and has been used to simulate ITG modes in LHD-like geometry [20]. Such modes are beyond the scope of the current implementation of the ideal MHD models, which do not include drift wave physics.

It is also of interest to simulate global modes in stellarators with low mode numbers, where the cancellation problem is particularly severe. We consider a Wendelstein 7-AS geometry, at varying values of normalised $\beta_{0}=\mu_{0} n_{0} T_{0} / B_{0}^{2}$. The iota profile is almost flat at approximately 0.4. A fixed numerical $\beta$ profile is applied with a maximum gradient at $s=0.5$. This case is run with the fully gyrokinetic mixed variables scheme, and the fluid hybrid model FLU-EUTERPE. Note that because no fast ion species is simulated, the physical model of CKA-EUTERPE is equivalent to the FLU-EUTERPE fluid hybrid model without gyrokinetic bulk ions.

In figure 4 left, the mode structure of a low-m global mode driven by the bulk $\beta$ gradient is plotted. The central plot shows the dependence of the growth rate of the mode on the normalised plasma $\beta$. It can be seen that there is a critical threshold in $\beta$ below which the mode is no longer present. These simulations were performed with the fluid hybrid model.

It is of interest whether this mode is suppressed by gyrokinetic effects. Furthermore, as it has not previously been observed, it is useful to verify that the mode is independently reproduced by different models. As such, one case was chosen and re-run with the mixed variables formulation of the full gyrokinetic model. Gyrokinetic effects introduce a finite real frequency, but the mode structure and evolution are otherwise comparable. In figure 4 right, the absolute value of the perturbed electrostatic potential from each model is plotted for the $\beta_{0}=1.8 \%$ case.

\section{Discussion and Conclusion}

In this article, a hierarchy of electromagnetic gyrokinetic and fluid hybrid models for simulating global modes has been described. More complex models incorporate more complete physics, but are more computationally demanding. In addition to developments to reduce the computational demands of full gyrokinetic simulations, one of which is described here, it remains useful to employ simpler, more numerically 

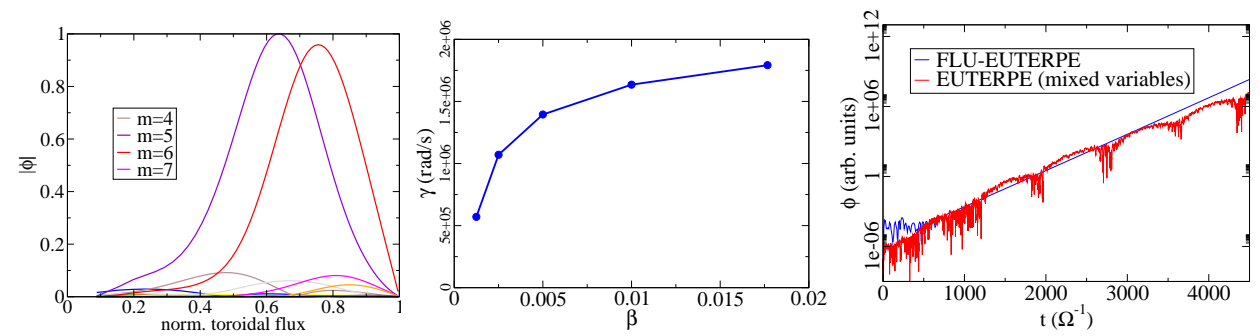

Figure 4. From left to right: the mode structure of the bulk pressure-driven mode in W7-AS geometry in terms of the poloidal harmonics; the linear growth rate of the mode as it varies with $\beta$; the evolution of the absolute value of the perturbed potential verified between the fluid and gyrokinetic models. Note that here $\beta_{0}=\mu_{0} n_{0} T_{0} / B_{0}^{2}$

robust models.

The fully gyrokinetic approach implemented in the code EUTERPE has been described. The mixed-variable formulation for the gyrokinetic equations of motion has been explained. The means by which this formulation may reduce the computational requirements of gyrokinetic simulations, including mitigation of the cancellation problem and simplification of the parallel dynamics, have been discussed. The fluidelectron, gyrokinetic-ion model FLU-EUTERPE has been detailed. This model avoids the cancellation problem entirely, at the expense of neglecting some physical effects. Finally, the hybrid MHD-gyrokinetic model CKA-EUTERPE has been described, which calculates power transfer between a gyrokinetic ion species and a fixed mode structure from an MHD code.

The uses and limits of these models were then considered. In simple cases, such as the low shear TAE case, all models were applicable, with each reduced model showing a stepwise order of magnitude reduction in computational requirement. In more complex cases, particularly where fast particles significantly modify the mode structure, the perturbative hybrid model is no longer sufficient. The fluid-electron, gyrokineticion model remains valid longer, but still diverges in some regimes, particularly where electron kinetics are important or the $E_{\|}$approximation is limiting. The mixed-variable formulation, meanwhile, was seen to permit efficient simulations with parameters that were previously challenging, such as low $m$ global modes in stellarator geometry, and its completeness at finite $E_{\|}$was demonstrated. Verification against this complete model then permitted more resource efficient parameter scans using the reduced models, using which the physics of a low $m$ electromagnetic stellarator mode were considered.

\section{Acknowledgments}

The authors wish to thank Tamás Fehér, Alessandro Zocco, Marco Restelli, and Robert Wolf for useful discussions and communications. The authors gratefully acknowledge the support of Per Helander. This work was carried out using the HELIOS supercomputer system at the Computational Simulation Centre of the International Fusion Energy Research (IFERC-CSC), Aomori, Japan, under the Broader Approach collaboration between Euratom and Japan, implemened by Fusion 
for Energy and JAEA. Simulations were also performed on the local cluster in Greifswald, for which the assistance of H. Leyh is appreciated. This project has received funding from the European Union's Horizon 2020 research and innovation programme under grant agreement number 633053. The views and opinions expressed herein do not necessarily reflect those of the European Commission. This work has been supported by EUROfusion Enabling Research CFP - WP14 - ER - 011ENEA Frascati - 01.

\section{References}

[1] Fasoli A et al. 2007 Nucl. Fusion 47 S264.

[2] Wesson J 1997 Tokamaks.

[3] Hahm T S, Lee W W and Brizard A J 1988 Phys. Fluids 311940.

[4] Lauber Ph, Günter S, Könies A, Pinches S D 2007 J. Comput. Physics 226(1) 447-465.

[5] Qin H 1998 Gyrokinetic Theory and Computational Methods for Electromagnetic Peturbations in Tokamaks, PhD thesis.

[6] Briguglio S, Vlad G, Zonca F and Kar C 1995 Phys. Plasmas 23711.

[7] Todo Y and Sato T 1998 Phys. Plasmas 5 1321-1327.

[8] Fu G Y et al. 2006 Phys. Plasmas 13052517.

[9] Gorelenkov N N, Cheng C Z, Fu G Y 1999 Phys. Plasmas 62802.

[10] Könies A, Mishchenko A and Hatzky R 2008 Theory of Fusion Plasmas 133-143.

[11] Spong D, D'Azevedo E and Todo Y 2010 Contr. Plasma Phys. 50 708-712.

[12] Cooper W A et al. 2011 Plasma Phys. Control. Fusion 53024001.

[13] Chen Y and Parker S 2000 Phys. Plasmas 8441.

[14] Lin Z and Chen L 2001 Phys. Plasmas 81447.

[15] Fehér T 2014 Simulation of the interaction between Alfvén waves and fast particles, $\mathrm{PhD}$ thesis.

[16] Kornilov V et al. 2004 Phys. Plasmas 113196.

[17] Chen Y and Parker S 2003 J. Comput. Phys. 189463.

18] Mishchenko A, Hatzky R and Könies A 2004 Phys. Plasmas 115480.

[19] Hatzky R, Könies A, Mishchenko A 2007 J. Comput. Phys. 225 568-590

[20] Mishchenko A, Cole M, Kleiber R and Könies A 2014 Phys. Plasmas 21052113.

[21] Mishchenko A, Könies A, Kleiber R and Cole M 2014 Phys. Plasmas 21092110.

[22] Chen Y and Parker S 2001 Phys. Plasmas 8441.

[23] Cole M et al. 2014 Phys. Plasmas 21072123.

[24] Könies A et al. 2012 Proc. of 24th Fusion Energy Conf., IAEA, Vienna.

[25] Berk H L, Breizman B N, Huanchun Ye 1992 Phys. Rev. Lett. 683563.

[26] Breizman B N and Sharapov S E 1995 Plasma Phys. Control. Fusion 371057

[27] Gorelenkov N N, Cheng C Z and Fu G Y 1999 Phys. Plasmas 62802.

[28] Mishchenko A, Könies A and Hatzky R 2014 Phys. Plasmas 21052114.

[29] Mishchenko A et al. 2014 Nucl. Fusion 54104003. 